\title{
Calculated cardiovascular risk after conversion from calcineurin inhibitor to belatacept in kidney transplant recipients: A randomized controlled trial
}

Obbo W. Bredewold ( $\sim$ o.W.Bredewold@lumc.nl)

Leiden University Medical Center Leiden

Joe Chan

Akershus University Hospital

My Svensson

Akershus University Hospital

Annette Bruchfeld

Linköping University

Johan W. Fijter

Leiden University Medical Center Leiden

Hans Furuland

Uppsala University Hospital

Josep M. Grinyo

Faculty of medicine and health sciences, University of Barcelona, Barcelona

Anders Hartmann

Oslo University Hospital

Hallvard Holdaas

Oslo University Hospital

Olof Hellberg

Örebro University

Alan Jardine

University of Glasgow

Lars Mjörnstedt

Sahlgrenska University Hospital

Karin Skov

Aarhus University Hospital

Knut T. Smerud

Smerud Medical Research International AS

Inga Soveri

Uppsala University Hospital

Sören S. Sörensen 
Copenhagen University Hospital

Anton-Jan Zonneveld

Leiden University Medical Center Leiden

\section{Bengt Fellstrom}

Uppsala University Hospital

\section{Research Article}

Keywords:

Posted Date: February 3rd, 2022

DOl: https://doi.org/10.21203/rs.3.rs-1283060/v1

License: (c) (i) This work is licensed under a Creative Commons Attribution 4.0 International License. Read Full License 


\section{Abstract}

In renal transplant recipients (RTRs), a belatacept-based immunosuppressive regimen is associated with beneficial effects on cardiovascular (CV) risk factors compared with calcineurin inhibitor (CNI)-based regimens. The aim of this randomized, multi-national trial was to compare calculated $\mathrm{CV}$ risk between belatacept and $\mathrm{CNI}$ (predominantly tacrolimus) treatments using a valdidated model developed for RTRs. From 9 transplant centers, RTRs from 3 to 60 months post-transplantation were recruited to either continue treatment with a $\mathrm{CNI}$-based regimen or switch to belatacept. We compared the change in estimated 7-year risk of major adverse cardiovascular events (MACE) and all-cause mortality after 12 months of treatment. In the 105 RTRs randomized, we found no differences between the treatment groups in predicted risk for MACE or mortality. Diastolic blood pressure was lower after belatacept treatment compared with CNI. The mean changes in traditional CV risk factors, including renal transplant function, were otherwise similar in both treatment groups. The belatacept group had four acute rejection episodes; two were severe rejections, of which one led to graft loss. In conclusion, we found no effects on calculated $\mathrm{CV}$ risk by switching to belatacept treatment.

\section{Introduction}

The risk of cardiovascular disease (CVD) in patients with renal failure is much higher than in the general population across all age groups ${ }^{1,2}$. While a successful transplant reduces this risk significantly, renal transplant recipients (RTRs) still have an annual cardiovascular (CV) event rate of $3.5-5 \%{ }^{3}$. Accordingly, CVD remains one of the leading causes of death in RTRs ${ }^{4,5}$. Managing a transplanted patient should therefore include $\mathrm{CV}$ risk reduction measures to improve both graft and patient outcomes. Current guidelines for prevention of CVD are based upon data from the general population and from studies specifically targeting CVD in RTRs ${ }^{6}$. In addition to addressing traditional risk factors for CVD, such as lifestyle choices, hypertension, hyperlipidemia, and diabetes, RTRs present two potentially modifiable factors: renal graft function and type of immunosuppressive maintenance regimen.

First, evidence indicates that declining graft function and graft loss are potentially modifiable risk factors for CVD and all-cause mortality in this population, which make strategies for optimizing graft function important ${ }^{7,8}$. Second, among immunosuppressive drugs used for transplantation, both steroids and calcineurin inhibitors (CNIs) are associated with adverse $\mathrm{CV}$ side effects ${ }^{9}$. Therefore, attempts have been made to minimize or eliminate their use. While these have led to reasonably safe steroid-free regimens 10-12, CNls are still the cornerstone of immunosuppression in modern solid organ transplantation. Early graft survival improved greatly after the introduction of cyclosporine (CsA) in the early $1980 \mathrm{~s}^{13}$, and tacrolimus (TAC) has been the CNI of choice since the $1990 \mathrm{~s}^{14}$. Despite the benefits of CNIs in the early post-transplant period, they have dose-dependent side effects, including post-transplant diabetes mellitus (PTDM), hypertension, hypercholesterolemia, and nephrotoxicity, leading to progressive decline in renal graft function ${ }^{15-19}$. Therefore, there is an ongoing incentive for development of novel immunosuppressive agents without the side effects of CNIs. 
Belatacept, a modified form of CTLA4-Ig, binds to CD80 and CD86 on antigen presenting cells, thus blocking CD28 mediated co-stimulation of T-cells. The BENEFIT trials have shown promise for belatacept as an option in designing a more favorable immunosuppressive regimen ${ }^{20-24}$. In brief, despite higher rates of early rejection, the relative risk of death or graft loss after 7 years was reduced by $43 \%$ in patients treated with belatacept versus CsA-treated patients, and eGFR in the belatacept-group was on average 22 $\mathrm{ml} / \mathrm{min} / 1.73 \mathrm{~m}^{2}$ higher than in the CsA-group. Furthermore, in a meta-analysis comparing belatacept with CNIs, treatment with belatacept was associated with lower blood pressure, lower incidence of diabetes and a more favourable lipid profile ${ }^{25}$.

However, it is not yet proven whether these findings translate into overall CVD reduction. Soveri et al. have previously developed a risk calculator for CVD and all-cause mortality for use in RTRs ${ }^{26}$. The group later used the data of the BENEFIT and BENEFIT-EXT trials to calculate the potential benefit associated with belatacept treatment and found a substantial calculated 7-year risk reduction for major adverse cardiac endpoints (MACE) and mortality by converting from CsA to belatacept ${ }^{27}$.

A shortcoming of belatacept that has hindered its implementation in kidney transplantation has been the relatively high rate of early rejection, as well as the lack of studies comparing its efficacy with low-dose TAC, the current standard of care in RTRs. In the present study, our aim was to investigate 1) the effects of conversion from a low-dose CNI-based therapy to belatacept on estimated risk of CVD and all-cause mortality using a previously validated calculator and 2) the changes in traditional markers of cardiovascular health, as well as measures of arterial stiffness.

\section{Results}

\subsection{Study participants and characteristics}

A total of 112 patients from 9 centers signed the patient informed consent form. Of these, one patient was a screen failure (history of rejection) and was never randomized. Of the 111 randomized patients, 6 withdrew consent before any study drug was given, 4 in the belatacept arm and 2 in the CNI arm. Thus, 105 patients were administered study medication: 54 in the belatacept arm and 51 in the CNI arm (defining our ITT population). In the belatacept-arm 5 patients were withdrawn from the study; 3 due to adverse events (AEs), 1 withdrew consent and 1 moved out of the country. Similarly, there were 2 withdrawals in the $\mathrm{CNI}$ arm; 1 due to $\mathrm{AE}$ and 1 withdrew consent. The remaining 49 patients in each treatment arm were defined as the per protocol (PP) population (Figure 1). As the difference between the PP population and the ITT population was quite small, we did not perform PP analyses to avoid the risk of type I error caused by multiple comparisons. The first patient was enrolled September 18th, 2014, and the last patient completed the study on September 13th, 2018. Baseline demographic data and clinical characteristics for each group are presented in Table 1. 
Table 1

Baseline demographics and clinical characteristics (ITT population).

\begin{tabular}{|c|c|c|}
\hline & Belatacept arm $(n=54)$ & CNI arm $(n=51)$ \\
\hline Female & $13(24 \%)$ & $13(25 \%)$ \\
\hline Age, years & $55.0(15.2)$ & $54.2(13.8)$ \\
\hline $\mathrm{BMI}, \mathrm{kg} / \mathrm{m}^{2}$ & $26.1(4.1)$ & $27.1(4.1)$ \\
\hline \multicolumn{3}{|l|}{ Renal replacement therapy } \\
\hline Number of transplantations & $1(1-2)$ & $1(1-2)$ \\
\hline Time since last transplantation, months & $25.3(3.7-59.6)$ & $23.4(3.1-58.8)$ \\
\hline Total time on renal replacement therapy, months & $35.6(12.1-489.5)$ & $36.8(5.3-220.8)$ \\
\hline \multicolumn{3}{|l|}{ Prior immunosuppressive therapy } \\
\hline Tacrolimus & $53(98 \%)$ & $48(94 \%)$ \\
\hline Cyclosporine & $1(2 \%)$ & $3(6 \%)$ \\
\hline Steroids & $50(93 \%)$ & $50(98 \%)$ \\
\hline Mycophenolate & $50(93 \%)$ & $47(92 \%)$ \\
\hline mTOR inhibitor & $3(6 \%)$ & $1(2 \%)$ \\
\hline \multicolumn{3}{|l|}{ Baseline immunosuppression trough levels } \\
\hline Tacrolimus & $5.8(1.7)$ & $5.7(1.7)$ \\
\hline Cyclosporine & 94 & $89(5.1)$ \\
\hline Diabetes mellitus & $12(22 \%)$ & $7(14 \%)$ \\
\hline Hypertension & $30(56 \%)$ & $33(65 \%)$ \\
\hline Systolic blood pressure, $\mathrm{mmHg}$ & $137(17.2)$ & $133(18.4)$ \\
\hline Diastolic blood pressure, $\mathrm{mmHg}$ & $84(9.7)$ & $81(11.2)$ \\
\hline \multicolumn{3}{|l|}{ Smoking habits } \\
\hline Current smoker & $6(11 \%)$ & $8(16 \%)$ \\
\hline Previous smoker & $19(35 \%)$ & $22(43 \%)$ \\
\hline \multicolumn{3}{|l|}{ Cardiovascular disease } \\
\hline Peripheral vascular disease & $8(15 \%)$ & $7(14 \%)$ \\
\hline Previous heart failure & $2(4 \%)$ & $3(6 \%)$ \\
\hline
\end{tabular}




\begin{tabular}{|lll|}
\hline & Belatacept arm (n=54) & CNI arm (n=51) \\
\hline Previous coronary heart disease & $4(7 \%)$ & $6(12 \%)$ \\
\hline Previous cerebrovascular disease & $2(4 \%)$ & $4(8 \%)$ \\
\hline Plasma creatinine, $\mu \mathrm{mol} / \mathrm{L}$ & $135(35.7)$ & $125(42.6)$ \\
\hline eGFR (mL/min/1.73 m²) & $49.4(14.8)$ & $56.6(19.1)$ \\
\hline hs-CRP, mg/L & $3.1(4.1)$ & $2.8(2.8)$ \\
\hline Plasma glucose, mmol/L & $6.2(1.8)$ & $5.9(1.7)$ \\
\hline Total cholesterol, $\mathrm{mmol} / \mathrm{L}$ & $5.0(1.0)$ & $1.6(0.6)$ \\
\hline HDL-cholesterol, mmol/L & $1.5(0.5)$ & $2.7(0.9)$ \\
\hline LDL-cholesterol, mmol/L & $2.7(0.9)$ & $1.9(0.9)$ \\
\hline Triglycerides, mmol/L & $1.9(0.9)$ & $1.0(0.3)$ \\
\hline Apolipoprotein B, g/L & $1.0(0.4)$ & $1.5(0.4)$ \\
\hline Apolipoprotein A1, g/L & $1.4(0.3)$ & \\
\hline $\begin{array}{l}\text { Data are presented as number (percentage) for categorical data, as mean value (standard deviation) } \\
\text { for continuous variables and as median (min - max) for renal replacement therapy. }\end{array}$ \\
\hline 2.2 EStimated risk Of MACE and mortality & \\
\hline
\end{tabular}

The primary endpoint was the estimated 7-year risk of MACE and all-cause mortality per the risk calculator developed by Soveri et al. (Figure 2). After 12 months of treatment, there was no statistically significant difference between the treatment groups in terms of change in predicted risk, neither for MACE nor for mortality (Table 2). 
Table 2

Estimated 7-year risk of MACE and mortality.

\begin{tabular}{|c|c|c|c|c|c|c|}
\hline & & \multicolumn{2}{|c|}{ Belatacept arm } & \multicolumn{2}{|l|}{ CNI arm } & \multirow[t]{2}{*}{ Difference } \\
\hline & & Baseline & $\begin{array}{l}\text { End of } \\
\text { study }\end{array}$ & Baseline & $\begin{array}{l}\text { End of } \\
\text { study }\end{array}$ & \\
\hline MACE & Mean (SD) & $\begin{array}{l}0.15 \\
(0.13)\end{array}$ & $0.15(0.15)$ & $\begin{array}{l}0.14 \\
(0.14)\end{array}$ & $0.15(0.15)$ & \\
\hline \multicolumn{2}{|c|}{$\begin{array}{l}\text { Log mean risk change }[95 \% \\
\mathrm{Cl}]\end{array}$} & \multicolumn{2}{|c|}{$-2.31[-2.40,-2.23]$} & \multicolumn{2}{|c|}{$-2.25[-2.33,-2.16]$} & $\begin{array}{l}0.06[-0.06, \\
0.14]\end{array}$ \\
\hline Mortality & Mean (SD) & $\begin{array}{l}0.21 \\
(0.19)\end{array}$ & $0.23(0.20)$ & $\begin{array}{l}0.19 \\
(0.18)\end{array}$ & $0.21(0.19)$ & \\
\hline \multicolumn{2}{|c|}{$\begin{array}{l}\text { Log mean risk change }[95 \% \\
\mathrm{Cl}]\end{array}$} & \multicolumn{2}{|c|}{$-1.94[-1.96,-1.91]$} & \multicolumn{2}{|c|}{$-1.92[-1.94,-1.90]$} & $\begin{array}{l}0.02[-0.01, \\
0.05]\end{array}$ \\
\hline
\end{tabular}

In the belatacept-arm, mean (SD) estimated 7-year risk of MACE at baseline was 0.15 (0.13), and it remained unchanged after 1 year to $0.15(0.15)$. Similarly, the risk estimation for the $\mathrm{CNI}$ continuation arm was $0.14(0.14)$ at baseline and $0.15(0.15)$ after 1 year. After applying the ANCOVA models and adjusting for hospital centers, the log mean risk prediction decreased by 2.31 (95\% Cl: 2.23, 2.40) for the belataceptgroup, and 2.25 (95\% Cl: 2.16, 2.33) for the $\mathrm{CNI}$ group. The difference between interventions in log mean risk prediction for MACE was 0.06 (95\% Cl: - $0.06,0.14)$.

The estimated 7-year mortality risk in the belatacept-arm at baseline was $0.21(0.19)$, which increased non-significantly to $0.23(0.20)$ after 1 year. Correspondingly for the $\mathrm{CNI}$ continuation arm, the predicted risk of mortality was $0.19(0.18)$ at baseline and increased non-significantly to $0.21(0.19)$ after 1 year. After applying the ANCOVA models and adjusting for hospital centers, the log mean risk prediction decreased by 1.94 (95\% Cl: $1.91,1.96)$ for the belatacept-group, and $1.92(95 \% \mathrm{Cl}: 1.90,1.94)$ for the CNI group. The difference between interventions in log mean risk prediction for mortality was $0.02(95 \% \mathrm{Cl}$ : $-0.01,0.05)$. An overview of the variables used in the risk calculation is presented in Table 3. 
Table 3

Overview of variables composing estimated cardiovascular risk.

\begin{tabular}{|c|c|c|c|c|c|}
\hline \multirow[b]{2}{*}{ Risk calculator composite } & \multirow[b]{2}{*}{ Variable } & \multicolumn{2}{|c|}{ Belatacept arm } & \multicolumn{2}{|l|}{$\mathrm{CNI}$ arm } \\
\hline & & Baseline & $\begin{array}{l}\text { End of } \\
\text { study }\end{array}$ & Baseline & $\begin{array}{l}\text { End of } \\
\text { study }\end{array}$ \\
\hline \multirow[t]{6}{*}{$\begin{array}{l}\text { Common for MACE and } \\
\text { mortality }\end{array}$} & Age, years & $\begin{array}{l}54.5 \\
(15.2)\end{array}$ & $\begin{array}{l}55.5 \\
(15.2)\end{array}$ & $\begin{array}{l}53.8 \\
(13.7)\end{array}$ & $\begin{array}{l}54.8 \\
(13.7)\end{array}$ \\
\hline & Creatinine, $\mu \mathrm{mol} / \mathrm{L}$ & $\begin{array}{l}135.1 \\
(35.7)\end{array}$ & $\begin{array}{l}132.2 \\
(44.1)\end{array}$ & $\begin{array}{l}124.7 \\
(42.6)\end{array}$ & $\begin{array}{l}119.1 \\
(38.4)\end{array}$ \\
\hline & Diabetes mellitus & $\begin{array}{l}12 \\
(22.2 \%)\end{array}$ & $\begin{array}{l}12 \\
(22.2 \%)\end{array}$ & $7(13.7 \%)$ & $7(13.7 \%)$ \\
\hline & Coronary HD & $4(7.4 \%)$ & $4(7.4 \%)$ & $6(11.8 \%)$ & $6(11.8 \%)$ \\
\hline & Current smoker & $\begin{array}{l}6 \\
(11.1 \%)\end{array}$ & $6(11.1 \%)$ & $8(15.7 \%)$ & $8(15.7 \%)$ \\
\hline & Previous smoker & $\begin{array}{l}19 \\
(35.2 \%)\end{array}$ & $\begin{array}{l}19 \\
(35.2 \%)\end{array}$ & $\begin{array}{l}22 \\
(43.1 \%)\end{array}$ & $\begin{array}{l}22 \\
(43.1 \%)\end{array}$ \\
\hline \multirow[t]{3}{*}{ MACE only } & $\begin{array}{l}\text { LDL-cholesterol, } \\
\mathrm{mmol} / \mathrm{L}\end{array}$ & $2.7(0.9)$ & $2.6(1.0)$ & $2.7(0.9)$ & $2.6(0.8)$ \\
\hline & $\begin{array}{l}\text { Number of } \\
\text { transplants: } 1\end{array}$ & $\begin{array}{l}51 \\
(94.4 \%)\end{array}$ & $\begin{array}{l}51 \\
(94.4 \%)\end{array}$ & $\begin{array}{l}48 \\
(94.1 \%)\end{array}$ & $\begin{array}{l}48 \\
(94.1 \%)\end{array}$ \\
\hline & $\begin{array}{l}\text { Number of } \\
\text { transplants: } 2\end{array}$ & $3(5.6 \%)$ & $3(5.6 \%)$ & $3(5.9 \%)$ & $3(5.9 \%)$ \\
\hline Mortality only & $\begin{array}{l}\text { Total time RRT, } \\
\text { months }\end{array}$ & $\begin{array}{l}51.4 \\
(69.5)\end{array}$ & $\begin{array}{l}62.9 \\
(69.6)\end{array}$ & $\begin{array}{l}45.1 \\
(37.0)\end{array}$ & $\begin{array}{l}56.9 \\
(37.0)\end{array}$ \\
\hline
\end{tabular}

Subgroup analysis was also performed to investigate whether time since transplantation influenced the results in risk calculation. Treatment arms were divided upon the median time after transplantation, thus creating an early and late group (before and after 26 months). There was no difference between belatacept and CNI in calculated risk of MACE $(p=0.33)$ and mortality $(p=0.56)$ in the subgroups.

\subsection{Traditional CVD risk factors}

The changes in traditional CV biomarkers from baseline to end of study are presented in Table 4 . The mean changes were similar between the treatment groups, except for a significant difference in diastolic blood pressure, with lower levels after belatacept treatment compared with CNI. Systolic blood pressure showed a similar reduction, but the difference was not statistically significant. 
Table 4

Change from baseline for traditional CVD risk factors.

\begin{tabular}{|llll|}
\hline Risk factor & Belatacept arm & CNI arm & p-value \\
\hline Systolic BP, mmHg & $-3.3(21.3)$ & $2.9(14.2)$ & 0.09 \\
\hline Diastolic BP, mmHg & $-2.6(10.0)$ & $2.8(10.7)$ & 0.01 \\
\hline hs-CRP, mg/L & $4.64(19.9)$ & $0.81(4.7)$ & 0.19 \\
\hline Plasma glucose, mmol/L & $0.04(2.5)$ & $-0.06(2.0)$ & 0.83 \\
\hline eGFR, mL/min/1.73 m² & $1.40(7.9)$ & $0.73(7.7)$ & 0.67 \\
\hline Total cholesterol, mmol/L & $0.22(2.8)$ & $-0.09(0.8)$ & 0.45 \\
\hline HDL-cholesterol, mmol/L & $-0.10(0.3)$ & $-0.02(0.2)$ & 0.08 \\
\hline LDL-cholesterol, mmol/L & $-0.10(0.7)$ & $-0.05(0.6)$ & 0.71 \\
\hline Triglycerides, mmol/L & $-0.06(0.8)$ & $-0.05(0.7)$ & 0.96 \\
\hline Apolipoprotein B, g/L & $-0.09(0.3)$ & $-0.06(0.2)$ & 0.59 \\
\hline Apolipoprotein A1, g/L & $0.02(0.3)$ & $0.01(0.3)$ & 0.88 \\
\hline $\begin{array}{l}\text { Presented as mean (standard deviation). BP = blood pressure. hs-CRP = high-sensitivity C-reactive } \\
\text { protein. eGFR = estimated glomerular filtration rate. }\end{array}$ & & \\
\hline P-value results from two-sample t-tests. & & & \\
\hline
\end{tabular}

\subsection{Arterial stiffness}

Arterial stiffness was measured at baseline and at end of study using the SphygmoCor ${ }^{\circledR}$ method.

Compared with the $\mathrm{CNI}$ group, central diastolic pressure in patients of the belatacept group decreased by $6.55 \mathrm{mmHg}(95 \% \mathrm{Cl}: 1.83,11.27 ; \mathrm{p}=0.007)$ after one year of treatment. For central systolic pressures, the difference of $6.1 \mathrm{mmHg}$ between study groups (95\% Cl: $-0.11,12.34 ; \mathrm{p}=0.054)$ was borderline significant. There were no differences between the treatment arms in central pulse pressure, pulse wave velocity and augmentation index (Table 5). 
Table 5

Change from baseline in arterial stiffness variables.

\begin{tabular}{|llll|}
\hline Risk factor & Belatacept arm & CNI arm & p-value \\
\hline Augmentation Index, \% & $-1.26(10.6)$ & $1.04(10.9)$ & 0.33 \\
\hline Pulse Wave Velocity, cm/sec & $-0.44(1.9)$ & $0.1(3.0)$ & 0.34 \\
\hline Central systolic pressure, $\mathrm{mmHg}$ & $-4.45(15.3)$ & $1.65(13.7)$ & 0.054 \\
\hline Central diastolic pressure, mmHg & $-3.72(12.1)$ & $2.83(9.6)$ & 0.007 \\
\hline Central pulse pressure, mmHg & $-0.60(11.3)$ & $-0.37(10.1)$ & 0.92 \\
\hline Presented as mean (standard deviation). P-value results from two-sample t-tests. \\
\hline
\end{tabular}

\subsection{Cardiovascular events and patient survival}

During the one-year study period, there were no cardiovascular events observed (including cardiovascular death, non-fatal myocardial infarction, non-fatal stroke, hospitalization due to congestive heart failure or angina pectoris, or coronary intervention) or deaths in the study population.

\subsection{Safety evaluation}

All patients in both study groups reported at least one AE during the duration of the study (Table 6). The majority of the events were of mild severity and considered unrelated to study drug. More patients in the belatacept-group (53.7\% vs $21.6 \%$ ) reported AEs that were considered possibly or probably related to the intervention. Three patients in the belatacept-group and one patient in the CNI continuation group reported AEs that led to withdrawal from the study. Serious Adverse Events (SAEs) were reported by $29.6 \%$ of the patients in the belatacept-group compared with $15.7 \%$ in the CNI group. Patients allocated to the belatacept-group had more infections (Table 7). There was 1 case of incident cancer (lung cancer), which occurred in the belatacept-group. 
Table 6

Number and proportion of patients with adverse events

\begin{tabular}{|lllll|}
\hline & \multicolumn{2}{l}{ Belatacept arm } & \multicolumn{2}{l|}{ CNI arm } \\
\hline Any adverse event & $n$ & $\%$ & $n$ & $\%$ \\
\hline 1 adverse event & 54 & 100 & 51 & 100 \\
\hline$>1$ adverse events & 10 & 18.5 & 21 & 41.2 \\
\hline Any possibly or probably intervention-related adverse events & 29 & 53.7 & 11 & 21.6 \\
\hline Adverse events leading to withdrawal & 44 & 81.5 & 30 & 58.8 \\
\hline Serious adverse events & 3 & 5.6 & 1 & 2.0 \\
\hline - suspected acute rejection & 16 & 29.6 & 8 & 15.7 \\
\hline - biopsy-proven acute rejection & 7 & 13.0 & 1 & 2.0 \\
\hline - graft loss due to acute rejection & 4 & 7.4 & 1 & 2.0 \\
\hline - cancer & 1 & 1.9 & 0 & - \\
\hline
\end{tabular}


Table 7

Adverse events reported by $\geq 5 \%$ of patients in either treatment group

\begin{tabular}{|lll|}
\hline Event & Belatacept arm (n=54) & CNI arm (n=51) \\
\hline Urinary tract infection & 35.3 & 7.8 \\
\hline Pyrexia & 31.5 & 2.0 \\
\hline Abdominal pain/discomfort & 18.6 & 2.0 \\
\hline Nasopharyngitis & 18.5 & 15.7 \\
\hline Respiratory tract infection & 14.9 & 9.8 \\
\hline Coughing & 14.8 & 2.0 \\
\hline Oedema & 9.4 & 6.0 \\
\hline Diarrhoea & 9.3 & 3.9 \\
\hline Anemia & 9.3 & 2.0 \\
\hline Fatigue & 7.4 & 2.0 \\
\hline Headache & 7.4 & 2.0 \\
\hline Dizziness & 7.4 & 0 \\
\hline Arthralgia & 3.7 & 5.9 \\
\hline Gastroenteritis & 1.0 & 5.9 \\
\hline Nausea & 5.6 & 2.0 \\
\hline Herpes zoster & 5.6 & 2.0 \\
\hline Myalgia & 5.6 & 0 \\
\hline Aphthous ulcer & 5.6 & \\
\hline Given as incidence rates (in \%) & & \\
\hline 8 & & 2.0 \\
\hline
\end{tabular}

During the study, 8 acute rejection episodes were suspected, and graft biopsies were obtained for further investigation. Acute rejection was confirmed in 4 of the 7 suspected cases in the belatacept-group, and in the single case in the $\mathrm{CNI}$ group. Three of the rejection episodes were considered severe (Banff grade IIA or higher): two in the belatacept-group and in the $\mathrm{CNI}$-treated patient. One patient (belatacept) proved refractory despite anti-rejection treatment with methylprednisolone and T-cell depleting antibody. All other rejection episodes recovered upon treatment with corticosteroids or anti-thymocyte globulin as per local practices.

\section{Discussion}


In this randomized study, where stable renal transplant patients were converted from a CNI-based maintenance immunosuppressive regimen to belatacept, no difference in calculated 7-year risk of MACE or all-cause mortality could be demonstrated after 1 year of follow-up. We were unable to find a significant effect on any of the three modifiable cardiovascular risk factors which were used as inputvariables in the risk calculator (serum LDL-cholesterol, diabetes-prevalence, and serum creatinine). The belatacept arm had significantly lower diastolic blood pressure, measured both centrally (SphygmoCor ${ }^{\circledR}$ method) and peripherally. We found a similar improvement for systolic pressure (Table 4, 5), but this difference was not statistically significant.

Of the three modifiable risk factors in the calculator, the expectations regarding effect on lipid profile were limited. While CsA has been implicated in dyslipidemia ${ }^{28}$, TAC seems to be less detrimental to lipid status. In our study, $94 \%$ of the participants were on TAC before randomization. Ferguson et al. ${ }^{29}$ compared three steroid-avoiding regimens of immunosuppression: belatacept with MMF vs belatacept with sirolimus vs TAC with MMF. Both belatacept-arms had lower LDL $(23.9 \mathrm{mg} / \mathrm{ml}$ and $25.0 \mathrm{mg} / \mathrm{ml}$ vs $34.0 \mathrm{mg} / \mathrm{ml}$ for TAC with MMF) after one year, but the difference was non-significant, possibly related to the limited sample-size of the study. Another observational study focusing on the metabolic effects of conversion from TAC to belatacept found improvement in GFR and acid-base status, but not in blood lipids ${ }^{30}$. Our findings are in line with these reports, as we found no effect on LDL-cholesterol (Table 3).

However, we are surprised by the lack of effect on GFR, which is in contrast to the BENEFIT studies, as well as other conversion studies reported in the literature ${ }^{24,31-33}$. In those studies, there was a consistent improvement in graft function by converting to belatacept. One possible explanation for this was the predominant use of TAC by our study participants with relatively low trough levels (Table 1) at baseline. In the Symphony trial ${ }^{34}$, the low-dose TAC group had an average trough-level of $6.7 \mathrm{ng} / \mathrm{ml} 1$ year after transplantation and achieved an eGFR on average $5.7 \mathrm{ml} / \mathrm{min} / 1.73 \mathrm{~m}^{2}$ higher than the other 3 comparator groups. A belatacept conversion study by Grinyo et al. ${ }^{35}$ examined 173 patients with a mean time after transplantation to randomization of 19 months, baseline eGFR of $54 \mathrm{ml} / \mathrm{min} / 1.73 \mathrm{~m}^{2}$, and a low immunologic risk profile, making the population reasonably comparable to ours. Belatacept patients in that study showed an average improvement in eGFR of $4.9 \mathrm{ml} / \mathrm{m} / 1.73 \mathrm{~m}^{2}$ compared to CNI-patients. At baseline, patients using TAC (56\%) had an average trough level of $7.2 \mathrm{ng} / \mathrm{mL}$, while patients on CsA (44\%) had an average trough level of $160.2 \mathrm{ng} / \mathrm{mL}$. In our study, the mean trough levels of TAC (5.7 $\mathrm{ng} / \mathrm{ml}$ ) and CsA (91 ng/ml, 4 patients only) at the time of randomization were lower compared to both these studies ${ }^{34,35}$. The lower $\mathrm{CNI}$ trough levels may have already significantly decreased the nephrotoxic side-effects and explain why our belatacept patients only experienced a non-significant gain in eGFR of $0.7 \mathrm{ml} / \mathrm{m} / 1.73 \mathrm{~m}^{2}$.

The third element of the calculator is the diabetes status. Multiple studies have corroborated the diabetogenicity of TAC in transplantation ${ }^{36-38}$. Furthermore, reversibility of beta cell dysfunction and of PTDM after TAC-withdrawal has been established in both animal studies and in clinical experience ${ }^{39-42}$. Thus, we expected to improve glycemic metabolism in converting from TAC to belatacept. However, no 
subject in our study reversed diabetes mellitus or developed PTDM in either study arm (Table 3). Also, triglycerides, serum ApoB, and serum ApoA1 did not improve (Table 4), which is of interest, since all three of these parameters are mentioned as risk factor for developing PTDM. ${ }^{43,44}$

Beside trough levels, we also need to consider another bias. All patients were already treated with CNI for a median of 26 months since transplantation. Serious negative side effects of $\mathrm{CNI}$-treatment could be less likely found in the control group, as patients suffering from these side effects could have been converted to alternative immunosuppression earlier on and thus not be eligible for this study.

The only positive effect that we found for belatacept was a significant improvement in diastolic blood pressure, measured both centrally (SphygmoCor ${ }^{\circledR}$ method) and peripherally. For systolic pressure, a similar improvement was found (Table 4), but it was not statistically significant ( $p=0.09$ ), most likely due to the relatively small sample size of this study. Although not included in the calculator, blood pressure is of course an established risk factor for cardiovascular disease. Moreover, high blood pressure is strongly associated with risk of graft failure and finding an improvement in this parameter could still indicate an advantage for belatacept-treatment ${ }^{45}$.

Regarding safety, AEs occurred in both groups, but SAEs were reported almost twice as often in belatacept-treated patients ( $29.6 \%$ vs $15.7 \%$ ), and the latter were more likely ( $5.6 \%$ vs $2.0 \%$ ) to discontinue their study treatment than patients treated with CNI. Rejection was seen more often in the belataceptpatients. Four episodes of biopsy-proven acute rejections occurred in the belatacept-group vs one single episode in the CNI-group (7.4\% vs $2.0 \%$ ). Three patients showed signs of vascular inflammation in the biopsy, corresponding to Banff grade II, two of which were in the belatacept-group. All three patients were treated according to local protocol with high-dose steroids (4) and T-cell depleting antibodies (1), despite which one belatacept-patient suffered graft loss and re-initiated dialysis treatment. The other two recovered with treatment.

The rate of rejection in this study is in line with earlier reports. For example, in the trial by Grinyo et al. ${ }^{35}$, $7.1 \%$ of belatacept-patients experienced rejection versus none in the CNI-group. In another trial by Adams et al. ${ }^{46}, 1$-year rejection rates were around $50 \%$ when belatacept was used right after transplantation, declining to $33 \%$ when TAC was tapered off 3-5 months after transplantation. When TAC was tapered after 11 months, the rejection rates between TAC- and belatacept-treated patients were similar, around $16 \%$. Other reports have described varying $(0-11 \%)$ rates of rejection, but these are data from nonrandomized 'rescue'-settings after even longer time post-transplantation and are therefore not comparable with our results 47,48 .

Beside rejection, urinary tract infections (UTIs), nasopharyngitis and other respiratory tract infections (RTIs) were more often seen in the belatacept arm (Table 7). The present study's planned visits could have led to a bias in the reporting of uncomplicated infections, since a study visit was planned every month for belatacept-patients, instead of every 3 months for the CNI-continuation group. 
Not a single case of pneumocystis-jirovecii pneumonia, cytomegalovirus- (CMV), polyoma- or EBVassociated disease was seen in the belatacept-patients. Three cases of CMV-infection were seen in CNIpatients. Previous reports have been inconclusive on opportunistic infections (OPIs) in belatacepttreatment. The follow-up study to the first belatacept-conversion trial noted a slightly higher incidence of viral infection (11\% vs $14 \%)^{35}$. In a recent study by Bertrand e.a. 50 OPI's were noted in 453 patients treated with belatacept $(9.8 \%)^{49}$. In a multivariate analysis of that study, the authors concluded that patients with low GFR ( $<25 \mathrm{ml} / \mathrm{min}$ ) and patients converted early after transplantation (within six months) were more likely to develop OPIs.

There was one case of lung cancer in the belatacept group in the present study. Previous studies have not indicated a higher risk of malignancy in belatacept beyond post-transplant-lymphoproliferative disorder 24,35 .

A major strength of the current study is the international multicenter-approach, making it representative for European transplantation practice. However, this study also has important limitations which must be taken into account. The study duration of 1 year was most likely too short to reveal a significant difference in renal function between the two study groups. We have overestimated the potential reduction in MACE and mortality for patients that use low-dose TAC instead of CsA. Another limitation was the heterogeneous time from transplantation to trial enrollment, and the small number of patients on CsA and the relatively large span of eGFR also contributed to the heterogeneity. Patients with severely diminished graft function were less likely to benefit from conversion.

In conclusion, we have shown no effect on calculated cardiovascular risk or renal function in this study comparing late conversion to belatacept with continuation of CNI-based immunosuppression. We did show a significant difference in diastolic blood pressure. We re-confirmed the increased chance of rejection when converting to belatacept. After more than 10 years of clinical experience, the place of belatacept in kidney transplantation is still not fully established, but it may be an attractive option when patients suffer from significant side effects of $\mathrm{CNI}$, like nephrotoxicity or PTDM. However, it is hard to define a significant benefit of belatacept for patients that are doing well on low-dose TAC-based therapy without severe CNI-related side-effects. Further studies are needed to define the place of belatacept in kidney transplantation.

\section{Methods}

\subsection{Study design}

This was a prospective, randomized, open label, parallel group, investigator-initiated, international multicenter trial (EudraCT no. 2013-001178-20, registered at www.clinicaltrialsregister.eu on 29-10-2013). Patients were randomized in 1:1 ratio to either continue treatment with a $\mathrm{CNI}$-based regimen or to switch to belatacept for a study duration of 12 months. An open design was chosen since CNIs were given as tablets daily and belatacept was administered as infusion every four weeks. 
Patients were recruited from 9 transplant centers in Denmark, the Netherlands, Norway, and Sweden. RTRs aged 18-80 years with a stable graft function (estimated glomerular filtration rate [eGFR] >20 $\mathrm{mL} / \mathrm{min}$ per $1.73 \mathrm{~m}^{2}$ ), 3-60 months post-transplantation treated with TAC or CsA were eligible for inclusion. Patients were excluded if they were Epstein-Barr virus (EBV) IgG seronegative, had severe de novo or recurrent renal disease, had a history of vascular or antibody-mediated rejection in the present transplant or had a history of recent malignancy.

The study was approved by the Regional Ethical Review Board in Uppsala, Sweden, and subsequently by the local ethics committees of the other affiliated hospitals. Written informed consent was obtained from all patients, and the trial was performed in accordance with the Declaration of Helsinki and the principles of Good Clinical Practice.

\subsection{Study medication}

For patients randomized to the study arm with belatacept, the previous CNI treatment (TAC or CsA) was tapered in the initial period as follows: $100 \%$ on day 1 , to $70-80 \%$ on day 7 , to $40-60 \%$ on day $15,20-30 \%$ on day 23 and none on day 29 and beyond. Belatacept was dosed $5 \mathrm{mg} / \mathrm{kg}$ IV on day 1, 15, 29, 43, 57 and then every month thereafter in the 12-month study period (Figure 3). Patients randomized to the control group with continuation of CNI treatment were to maintain trough levels of CsA between 75 and 200 $\mathrm{ng} / \mathrm{ml}$ and TAC between 5 and $10 \mathrm{ng} / \mathrm{ml}$. Both groups were to continue their underlying immunosuppressive regimen, consisting of mycophenolate mofetil (MMF) or mammalian target of rapamycin inhibitor and corticosteroids. Any other concomitant medication necessary to maintain the patients' baseline condition or to treat a coexisting disease was permitted.

\subsection{Efficacy assessment and procedures}

The primary endpoint of this trial was estimated cardiovascular risk after 12 months, using a prediction model developed for RTRs by Soveri et al. ${ }^{26}$. The estimated 7-year risk of MACE and mortality in the two treatment groups were calculated as a linear combination of the following variables: age, previous coronary heart disease, previous smoker, current smoker, creatinine, diabetes mellitus, low-density lipoprotein (LDL), number of transplants and total time on renal replacement therapy (Figure 2). Secondary endpoints were arterial stiffness, traditional CVD risk factors in RTRs (blood pressure, lipid profiles and eGFR), acute rejections, allograft loss, CV events and patient survival. Blood samples were drawn at a fasting state in the morning at baseline and at end of study visits for measurement of renal function and CV biomarkers: creatinine, high-sensitivity C-reactive protein (hs-CRP), total cholesterol, highdensity lipoprotein cholesterol (HDL-cholesterol), LDL cholesterol, triglycerides, apolipoprotein B (ApoB) and apolipoprotein A1 (ApoA1). Arterial stiffness was assessed at the same time points by measuring central pulse pressure, pulse wave velocity and augmentation index using the SphygmoCorß method ${ }^{50}$.

\subsection{Sample size and randomization}

We performed a power calculation hypothesizing that the intervention arm would decrease the risk of MACE by $30 \%$. We came to that estimate by extrapolation of the reduction in calculated risk in the 
previously mentioned paper by Soveri e.a. ${ }^{27}$; the calculated risk of MACE for BENEFIT-patients decreased by $31.2 \%$ (from $14.3-10.9 \%$ ), and for mortality by $40 \%(17.5-12.5 \%)$. The corresponding risk reduction for BENEFIT-EXT-patients was $27.8 \%$ (22.5 to $17.6 \%)$ and $22.6 \%$ (30.9-25.2\%). For a two-sample t-test on a two-sided significance level of 0.05 , assuming a standard deviation of 0.64 (on the natural logarithmic scale), a sample size of 51 per group was required to obtain a power of $0.8(80 \%)$ to detect a $30 \%$ calculated risk reduction in MACE. The ANCOVA model was expected to have slightly greater power than the two-sample t-test, and therefore a sample size of 102 patients was seen as sufficient for this study. To account for $8 \%$ drop-out, a total of 110 patients, 55 per treatment arm were included in the study.

Randomization to treatment arm was performed using a computerized procedure, stratified by center, in a 1:1 ratio.

\subsection{Statistical analysis}

The primary endpoint was a comparison of the estimated CV risk between treatment groups (CNI- vs. belatacept-based immunosuppression) at one year. For patients who discontinued the study before one year, the last available estimate of CV risk was used in the analysis of the intention-to-treat (ITT) population. Due to a skewed distribution, estimated CV risk was log-transformed. The primary analysis was performed using analysis of covariance (ANCOVA) with treatment as a group variable and baseline log CV risk for MACE and center as covariates. All other comparisons on primary and secondary endpoints were based on ITT comparisons of treatment groups using two-sample t-test, or ANCOVA with correction for baseline variables and/or center. A two-sided $P$ value of $<0.05$ was considered statistically significant. Analyses were performed using SAS ${ }^{8}$ version 9.4 (SAS Institute, Cary, NC).

\section{Abbreviations}

$\mathrm{AE}$ adverse event

ANCOVA analysis of covariance

ApoA1 apolipoprotein A1

ApoB apolipoprotein B

CNI calcineurin inhibitor

CsA cyclosporine

CV cardiovascular

CVD cardiovascular disease

EBV Epstein-Barr virus

eGFR estimated glomerular filtration rate 
GFR glomerular filtration rate

HDL high-density lipoprotein

hs-CRP high-sensitivity C-reactive protein

ITT intention-to-treat

LDL low-density lipoprotein

MACE major adverse cardiovascular event

MMF mycophenolate mofetil

OPI opportunistic infection

PP per protocol

PTDM post-transplant diabetes mellitus

$\mathrm{RTI}$ respiratory tract infection

RTR renal transplant recipients

SAE serious adverse event

TAC tacrolimus

\section{Declarations}

\section{ACKNOWLEDGMENTS}

The trial has received a financial grant from Bristol-Myers Squibb. Smerud Medical Research International AS was responsible for administrative project management including monitoring, data management, statistical analyses and compilation of the full clinical study report. The authors wish to thank Andre Western as lead study monitor and Kristoffer Yunpeng Ding as lead statistician for their support.

\section{Study Steering committee:}

Prime principal investigator of each participating center; Hallvard Holdaas, Oslo; Alan Jardine, Glasgow; Bengt Fellström, Uppsala, chairman of SSC.

\section{Data and Safety Monitoring Board:}

Anders Hartmann, Oslo; Josep M Grinyo, Barcelona.

\section{Author contributions}


OWB, JC First draft of manuscript

OWB, JC, MS, JWdF, KTS, AjvZ, BF Substantial revision and finalization of manuscript

OWB, MS, AB, HF, AH, HH, OH, LM, KS, SSS, BF Inclusion of patients and acquisition of data.

JG, AJ, BF Conception of study

JWdF, JMG, HH, AJ, IS, AJvZ, BF Study design

OWB, JC, MS, JWdF, KTS, AJvZ, BF Analysis and data preparation

\section{Data availability statement.}

The data used to support the findings are included in the manuscript. Any additional (raw) data are available from the author (OWB) and co-author (KTS) upon reasonable request. Restrictions to availability may apply due to privacy or ethical reasons.

\section{Competing interests}

The authors of this manuscript have the following conflicts of interest to disclose:

MS has received honoraria from Astellas.

KTS reports that his employer, Smerud Medical Research International AS, is a contract research organisation that delivered clinical trial management services to this study and was remunerated by Bristol Myers Squibb for that work.

IS has received honoraria from Sandoz, Vifor and Astra Zeneca

BF has received funding or honoraria from Astra Zeneca, Novartis, Astellas, Alexion, Bristol Myers Squibb and Calliditas Therapeutics.

The other authors have no conflict of interests to disclose.

The investigational product, belatacept, was supplied free of charge by Bristol Myers Squibb.

\section{References}

1. Baigent, C., Burbury, K. \& Wheeler, D. Premature cardiovascular disease in chronic renal failure. Lancet 356, 147-152, doi:10.1016/S0140-6736(00)02456-9 (2000).

2. Jardine, A. G., Gaston, R. S., Fellstrom, B. C. \& Holdaas, H. Prevention of cardiovascular disease in adult recipients of kidney transplants. Lancet 378, 1419-1427, doi:10.1016/S0140-6736(11)61334-2 (2011). 
3. Kasiske, B. L. et al. KDIGO clinical practice guideline for the care of kidney transplant recipients: a summary. Kidney Int 77, 299-311, doi:10.1038/ki.2009.377 (2010).

4. Methven, S., Steenkamp, R. \& Fraser, S. UK Renal Registry 19th Annual Report: Chapter 5 Survival and Causes of Death in UK Adult Patients on Renal Replacement Therapy in 2015: National and Centrespecific Analyses. Nephron 137 Suppl 1, 117-150, doi:10.1159/000481367 (2017).

5. Saran, R. et al. US Renal Data System 2017 Annual Data Report: Epidemiology of Kidney Disease in the United States. Am J Kidney Dis 71, A7, doi:10.1053/j.ajkd.2018.01.002 (2018).

6. Holdaas, H. et al. Effect of fluvastatin on cardiac outcomes in renal transplant recipients: a multicentre, randomised, placebo-controlled trial. Lancet 361, 2024-2031, doi:10.1016/S01406736(03)13638-0 (2003).

7. Fellstrom, B. et al. Renal dysfunction as a risk factor for mortality and cardiovascular disease in renal transplantation: experience from the Assessment of Lescol in Renal Transplantation trial. Transplantation 79, 1160-1163, doi:10.1097/01.tp.0000160764.35083.b8 (2005).

8. Svensson, M., Jardine, A., Fellstrom, B. \& Holdaas, H. Prevention of cardiovascular disease after renal transplantation. Curr Opin Organ Transplant 17, 393-400, doi:10.1097/MOT.0b013e3283560a3b (2012).

9. Jardine, A. G. Assessing the relative risk of cardiovascular disease among renal transplant patients receiving tacrolimus or cyclosporine. Transpl Int 18, 379-384, doi:10.1111/j.14322277.2005.00080.x (2005).

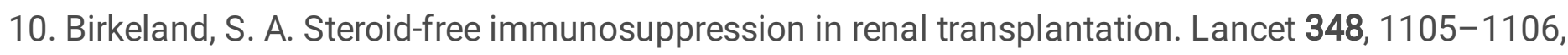
doi:10.1016/s0140-6736(05)64455-8 (1996).

11. Pathak, V. et al. Low-dose Rituximab and Thymoglobulin Induction With Steroid-free Maintenance Immunosuppression and Protocol Biopsies Improves Long-term Patient and Graft Survival After Kidney Transplantation: Survival and Safety Outcomes in More Than 1100 Patients From a Single Center. Transplant Direct 5, e475, doi:10.1097/TXD.0000000000000923 (2019).

12. Woodle, E. S. et al. A prospective, randomized, double-blind, placebo-controlled multicenter trial comparing early (7 day) corticosteroid cessation versus long-term, low-dose corticosteroid therapy. Ann Surg 248, 564-577, doi:10.1097/SLA.0b013e318187d1da (2008).

13. Hariharan, S. et al. Improved graft survival after renal transplantation in the United States, 1988 to 1996. N Engl J Med 342, 605-612, doi:10.1056/NEJM200003023420901 (2000).

14. Ong, S. C. \& Gaston, R. S. Thirty Years of Tacrolimus in Clinical Practice. Transplantation Publish Ahead of Print, doi:10.1097/tp.0000000000003350 (2020).

15. Vincenti, F. et al. Results of an international, randomized trial comparing glucose metabolism disorders and outcome with cyclosporine versus tacrolimus. Am J Transplant 7, 1506-1514, doi:10.1111/j.1600-6143.2007.01749.x (2007).

16. Hoorn, E. J. et al. The calcineurin inhibitor tacrolimus activates the renal sodium chloride cotransporter to cause hypertension. Nat Med 17, 1304-1309, doi:10.1038/nm.2497 (2011). 
17. Heisel, O., Heisel, R., Balshaw, R. \& Keown, P. New onset diabetes mellitus in patients receiving calcineurin inhibitors: a systematic review and meta-analysis. Am J Transplant 4, 583-595, doi:10.1046/j.1600-6143.2003.00372.x (2004).

18. Nankivell, B. J., Chapman, J. R., Bonovas, G. \& Gruenewald, S. M. Oral cyclosporine but not tacrolimus reduces renal transplant blood flow. Transplantation 77, 1457-1459 (2004).

19. Nankivell, B. J. et al. The natural history of chronic allograft nephropathy. N Engl J Med 349, 23262333, doi:10.1056/NEJMoa020009 (2003).

20. Durrbach, A. et al. A phase III study of belatacept versus cyclosporine in kidney transplants from extended criteria donors (BENEFIT-EXT study). Am J Transplant 10, 547-557, doi:10.1111/j.16006143.2010.03016.x (2010).

21. Durrbach, A. et al. Long-Term Outcomes in Belatacept- Versus Cyclosporine-Treated Recipients of Extended Criteria Donor Kidneys: Final Results From BENEFIT-EXT, a Phase III Randomized Study. Am J Transplant 16, 3192-3201, doi:10.1111/ajt.13830 (2016).

22. Larsen, C. P. et al. Belatacept-based regimens versus a cyclosporine A-based regimen in kidney transplant recipients: 2-year results from the BENEFIT and BENEFIT-EXT studies. Transplantation 90, 1528-1535, doi:10.1097/TP.0b013e3181ff87cd (2010).

23. Vincenti, F. et al. A phase III study of belatacept-based immunosuppression regimens versus cyclosporine in renal transplant recipients (BENEFIT study). Am J Transplant 10, 535-546, doi:10.1111/j.1600-6143.2009.03005.x (2010).

24. Vincenti, F. et al. Belatacept and Long-Term Outcomes in Kidney Transplantation. N Engl J Med 374, 333-343, doi:10.1056/NEJMoa1506027 (2016).

25. Masson, P., Henderson, L., Chapman, J. R., Craig, J. C. \& Webster, A. C. Belatacept for kidney transplant recipients. The Cochrane database of systematic reviews, Cd010699, doi:10.1002/14651858.CD010699.pub2 (2014).

26. Soveri, I. et al. A cardiovascular risk calculator for renal transplant recipients. Transplantation $\mathbf{9 4}$, 57-62, doi:10.1097/TP.0b013e3182516cdc (2012).

27. Soveri, I. et al. The external validation of the cardiovascular risk equation for renal transplant recipients: applications to BENEFIT and BENEFIT-EXT trials. Transplantation 95, 142-147, doi:10.1097/TP.0b013e31827722c9 (2013).

28. Mathis, A. S., Dave, N., Knipp, G. T. \& Friedman, G. S. Drug-related dyslipidemia after renal transplantation. Am J Health Syst Pharm 61, 565-585; quiz 586-567 (2004).

29. Ferguson, R. et al. Immunosuppression with belatacept-based, corticosteroid-avoiding regimens in de novo kidney transplant recipients. Am J Transplant 11, 66-76, doi:10.1111/j.16006143.2010.03338.x (2011).

30. Schulte, K. et al. Late conversion from tacrolimus to a belatacept-based immuno-suppression regime in kidney transplant recipients improves renal function, acid-base derangement and mineral-bone metabolism. J Nephrol 30, 607-615, doi:10.1007/s40620-017-0411-0 (2017). 
31. Vanrenterghem, Y. et al. Belatacept-based regimens are associated with improved cardiovascular and metabolic risk factors compared with cyclosporine in kidney transplant recipients (BENEFIT and BENEFIT-EXT studies). Transplantation 91, 976-983, doi:10.1097/TP.0b013e31820c10eb (2011).

32. Florman, S. et al. Efficacy and Safety Outcomes of Extended Criteria Donor Kidneys by Subtype: Subgroup Analysis of BENEFIT-EXT at 7 Years After Transplant. Am J Transplant 17, 180-190, doi:10.1111/ajt.13886 (2017).

33. Poster Abstracts. 20, 539-1166, doi:https://doi.org/10.1111/ajt.16171 (2020).

34. Ekberg, H. et al. Reduced exposure to calcineurin inhibitors in renal transplantation. N Engl J Med 357, 2562-2575, doi:10.1056/NEJMoa067411 (2007).

35. Grinyó, J. M. et al. Safety and Efficacy Outcomes 3 Years After Switching to Belatacept From a Calcineurin Inhibitor in Kidney Transplant Recipients: Results From a Phase 2 Randomized Trial. Am J Kidney Dis 69, 587-594, doi:10.1053/j.ajkd.2016.09.021 (2017).

36. Fan, Y., Xiao, Y. B. \& Weng, Y. G. Tacrolimus versus cyclosporine for adult lung transplant recipients: a meta-analysis. Transplant Proc 41, 1821-1824, doi:10.1016/j.transproceed.2008.11.016 (2009).

37. Knoll, G. A. \& Bell, R. C. Tacrolimus versus cyclosporin for immunosuppression in renal transplantation: meta-analysis of randomised trials. BMJ 318, 1104-1107, doi:10.1136/bmj.318.7191.1104 (1999).

38. Penninga, L. et al. Tacrolimus versus cyclosporin as primary immunosuppression for lung transplant recipients. The Cochrane database of systematic reviews, CD008817, doi:10.1002/14651858.CD008817.pub2 (2013).

39. Dai, C. et al. Tacrolimus- and sirolimus-induced human beta cell dysfunction is reversible and preventable. JCl Insight 5, doi:10.1172/jci.insight.130770 (2020).

40. de Graav, G. N., van der Zwan, M., Baan, C. C., Janssen, J. \& Hesselink, D. A. Improved Glucose Tolerance in a Kidney Transplant Recipient With Type 2 Diabetes Mellitus After Switching From Tacrolimus To Belatacept: A Case Report and Review of Potential Mechanisms. Transplant Direct 4, e350, doi:10.1097/TXD.0000000000000767 (2018).

41. Terrec, F. et al. Late Conversion From Calcineurin Inhibitors to Belatacept in Kidney-Transplant Recipients Has a Significant Beneficial Impact on Glycemic Parameters. Transplant Direct 6, e517, doi:10.1097/TXD.0000000000000964 (2020).

42. Ghisdal, L. et al. Conversion from tacrolimus to cyclosporine A for new-onset diabetes after transplantation: a single-centre experience in renal transplanted patients and review of the literature. Transpl Int 21, 146-151, doi:10.1111/j.1432-2277.2007.00589.x (2008).

43. Malyala, R., Rapi, L., Nash, M. M. \& Prasad, G. V. R. Serum Apolipoprotein B and A1 Concentrations Predict Late-Onset Posttransplant Diabetes Mellitus in Prevalent Adult Kidney Transplant Recipients. Can J Kidney Health Dis 6, 2054358119850536, doi:10.1177/2054358119850536 (2019).

44. Guzman, G. E. et al. Risk Factors Related to New-Onset Diabetes after Renal Transplantation in Patients of a High Complexity University Hospital in Colombia, 20 Years of Experience. Int J Endocrinol 2020, 8297192, doi:10.1155/2020/8297192 (2020). 
45. Mangray, M. \& Vella, J. P. Hypertension after kidney transplant. Am J Kidney Dis 57, 331-341, doi:10.1053/j.ajkd.2010.10.048 (2011).

46. Adams, A. B. et al. Belatacept Combined With Transient Calcineurin Inhibitor Therapy Prevents Rejection and Promotes Improved Long-Term Renal Allograft Function. Am J Transplant 17, 29222936, doi:10.1111/ajt.14353 (2017).

47. Brakemeier, S. et al. Experience with belatacept rescue therapy in kidney transplant recipients. Transpl Int 29, 1184-1195, doi:10.1111/tri.12822 (2016).

48. Gupta, S., Rosales, I. \& Wojciechowski, D. Pilot Analysis of Late Conversion to Belatacept in Kidney Transplant Recipients for Biopsy-Proven Chronic Tacrolimus Toxicity. J Transplant 2018, 1968029, doi:10.1155/2018/1968029 (2018).

49. Bertrand, D. et al. Opportunistic Infections and Efficacy Following Conversion to Belatacept-Based Therapy after Kidney Transplantation: A French Multicenter Cohort. J Clin Med 9, doi:10.3390/jcm9113479 (2020).

50. Butlin, M. \& Qasem, A. Large Artery Stiffness Assessment Using SphygmoCor Technology. Pulse (Basel) 4, 180-192, doi:10.1159/000452448 (2017).

\section{Figures}




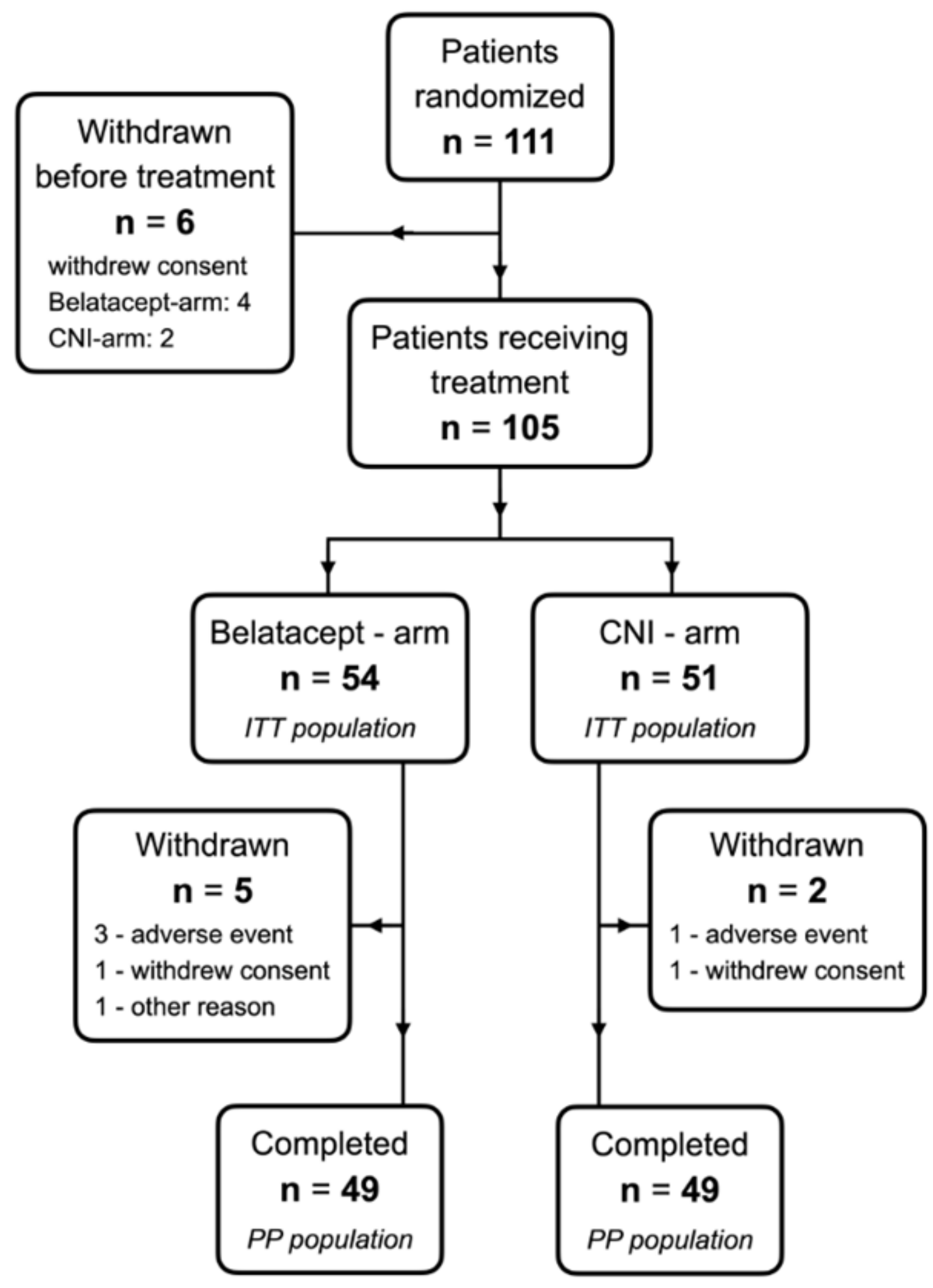

Figure 1

Study flow chart. 


\section{7-Year Risk Calculation of \\ Major Adverse Cardiac Event}

- Diabetes mellitus:

- LDL-cholesterol:

- Current smoker:

- Previous smoker:

- Coronary heart disease:

- Number of transplants:

- Creatinine:

- Age:

Yes / No

Yes / No

Yes / No

$\mu \mathrm{mol} / \mathrm{L}$

Years

\section{7-Year Risk Calculation of \\ Mortality}

- Diabetes mellitus: Yes */ No

- Current smoker:

- Previous smoker:

Yes / No

\#
- Coronary heart disease: Yes / No

- Total time on RRT: Months

- Creatinine: $\quad \mu \mathrm{mol} / \mathrm{L}$

- Age: Years

Figure 2

Cardiovascular risk calculator for renal transplant recipients (Soveri et al., 2012).

List of variables used in the cardiovascular risk calculator. *Includes post-transplant diabetes mellitus. RRT = renal replacement therapy (including dialysis and transplantation)

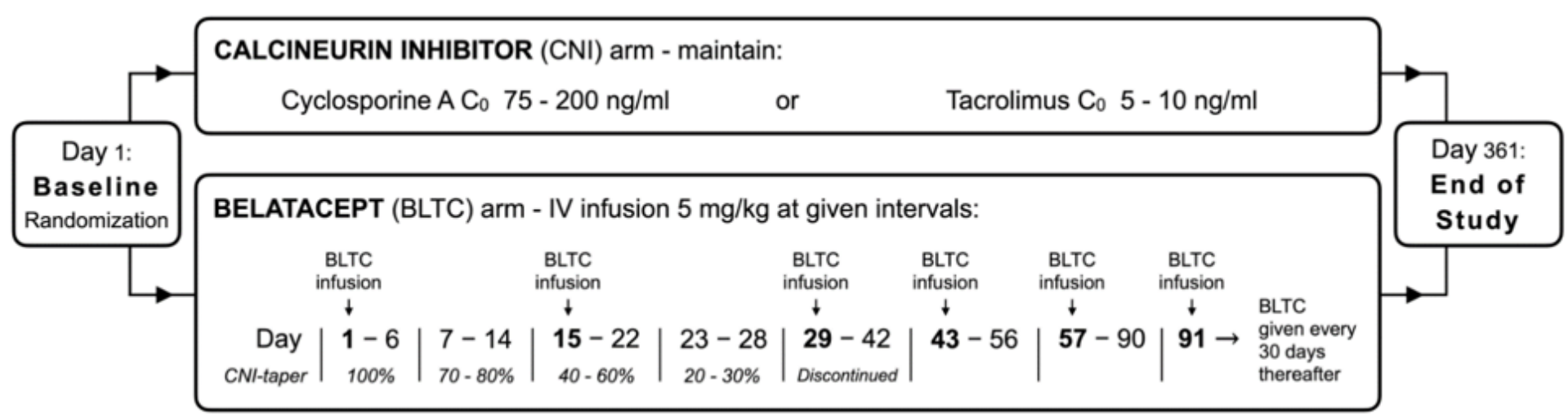

Figure 3

Conversion and dosing scheme. 